\title{
Initiating a practice change: Prescribing probiotics concurrently with antibiotic therapy
}

\author{
Patty Hermosilla \\ West Virginia University
}

Follow this and additional works at: https://researchrepository.wvu.edu/etd

\section{Recommended Citation}

Hermosilla, Patty, "Initiating a practice change: Prescribing probiotics concurrently with antibiotic therapy" (2009). Graduate Theses, Dissertations, and Problem Reports. 3507.

https://researchrepository.wvu.edu/etd/3507

This Dissertation is protected by copyright and/or related rights. It has been brought to you by the The Research Repository @ WVU with permission from the rights-holder(s). You are free to use this Dissertation in any way that is permitted by the copyright and related rights legislation that applies to your use. For other uses you must obtain permission from the rights-holder(s) directly, unless additional rights are indicated by a Creative Commons license in the record and/ or on the work itself. This Dissertation has been accepted for inclusion in WVU Graduate Theses, Dissertations, and Problem Reports collection by an authorized administrator of The Research Repository @ WVU.

For more information, please contact researchrepository@mail.wvu.edu. 


\title{
Initiating a Practice Change: Prescribing Probiotics Concurrently with Antibiotic Therapy
}

\section{Patty Hermosilla, MSN, FNP-BC}

Doctoral Research Project submitted to the School of Nursing at West Virginia University

in partial fulfillment of the requirements for the degree of

\author{
Doctor of Nursing Practice \\ Kari Sand-Jecklin, EdD, Chair \\ Harakh Dedhia, MD \\ Lisa Hardman, DNP \\ Morgantown, West Virginia \\ 2009
}

Keyword: probiotics, antibiotic associated diarrhea, education, clinical guidelines 


\section{ABSTRACT}

\section{Initiating a Practice Change: Prescribing Probiotics Concurrently with Antibiotic Therapy}

\section{Patty Hermosilla}

Background and Significance

Over half of all hospitalized patients are treated with antibiotics and antibiotic use is rising. There has been a 500\% increase in antibiotic associated diarrhea, a common side effect of antibiotic use, in the last decade. Probiotics are a safe and cost effective measure to prevent or reduce antibiotic associated diarrhea.

Problem Statement

The majority of patients admitted to the medical intensive care unit receive antibiotic therapy; however, few are prescribed probiotic therapy concurrent with antibiotics.

Project Design

Education was provided to health care providers regarding antibiotic associated diarrhea and the benefits of probiotic use. A guideline was developed to assist health care providers in ordering probiotics.

Evaluation Plan

Health care providers' knowledge, attitude, and beliefs, pre and post education intervention were evaluated. Probiotic prescribing rates were tracked pre and post intervention.

Results

There was a statistically significant increase in knowledge and a significant change in attitude after the education intervention. There was also a 2 fold increase in prescribing rates; however a very small number of probiotics were prescribed.

\section{Recommendations}

Attempt this practice change on a unit with a more stable staff and a non ICU population. Potentially use yogurt instead of a probiotic tablet in the practice guideline. Also, soliciting change champions may promote probiotic use. Using a flag in the medication ordering system to remind providers to order a probiotic or yogurt concurrently with antibiotic therapy may also increase probiotic prescription rates. Additional research at the institution is recommended to demonstrate the effectiveness of probiotics. 


\section{Acknowledgements}

I want to extend my sincere thanks and appreciation to Dr. Kari Sand-Jecklin, Chairperson of my committee, for her endless support, ingenious guidance, and most of all the precious commodity of time she gave me. I am especially grateful for her expertise and valuable assistance in the statistical analysis. I would also like to thank Dr. Harakh Dedhia, and Dr. Lisa Hardman for their furtherance, advice and time.

Furthermore, I would like to thank God for his blessings during those particularly difficult times. For my husband, Dennis, and children Nick, Ali, and Dominica, special thanks for your loving support, patience, and understanding throughout this educational endeavor. Moreover, I would like to thank my grandchild Aidan for making me laugh. Likewise, I would like to thank my father, William Smith, and my late mother, Dolores Smith, for giving me life, making it possible for me to actualize this effort. Lastly, thanks to my entire family for their unending support and encouragement. 


\section{Table of Contents}

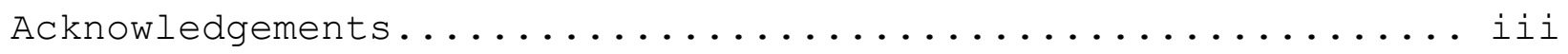

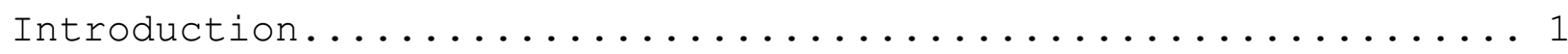

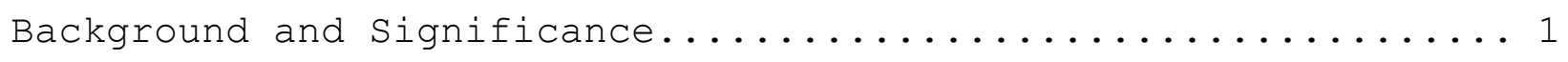

Significance of Probiotics.................... 5

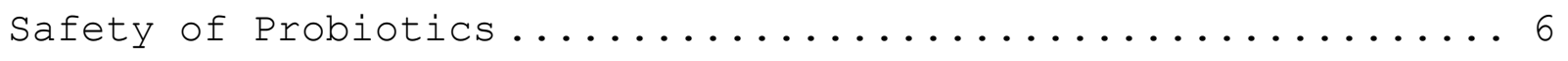

Probiotic Dose $\ldots \ldots \ldots \ldots \ldots \ldots \ldots \ldots \ldots \ldots \ldots \ldots \ldots \ldots$

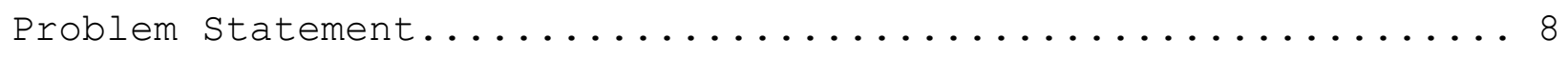

Theoretical Framework........................ 9

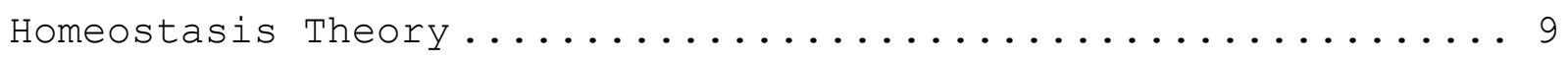

Change Theory .................................. 10

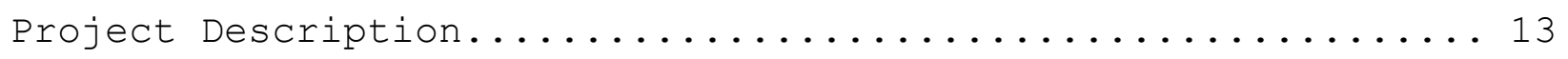

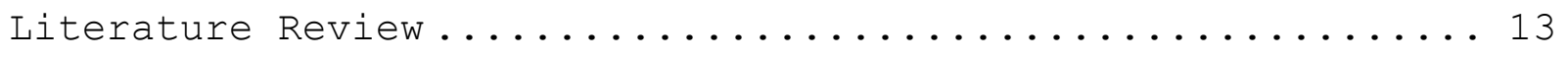

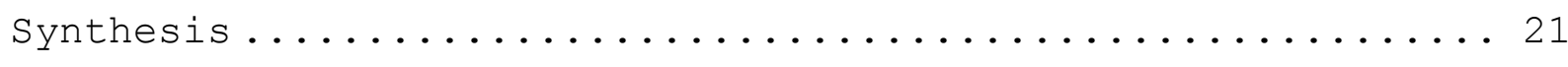

Congruence of Organizations Strategic Plan ........... 22

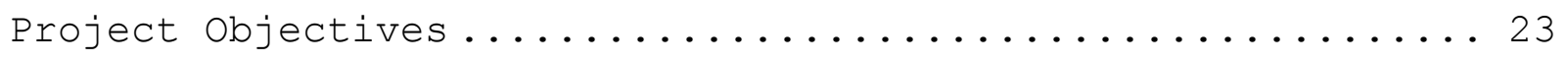

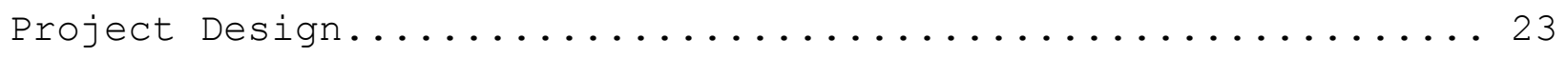




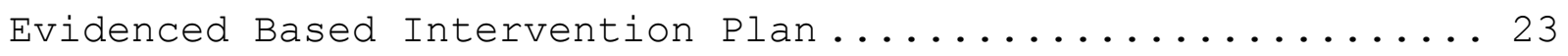

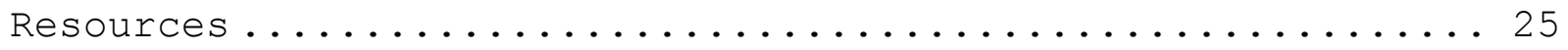

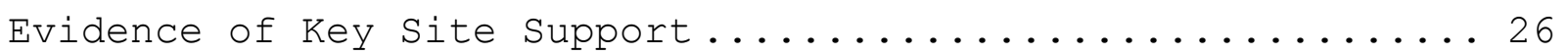

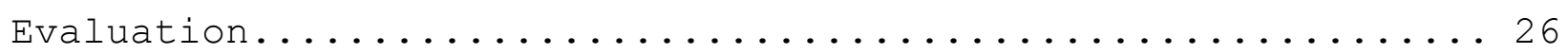

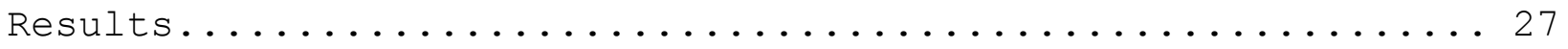

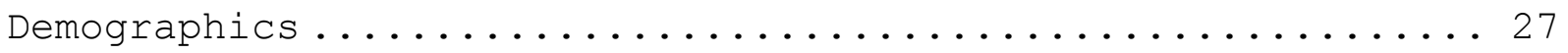

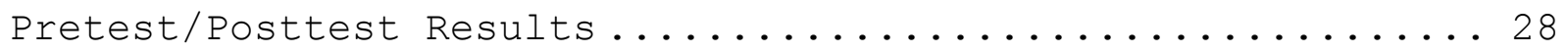

Table 1: Knowledge Items: Percent Correct Pre and Post Test... 29

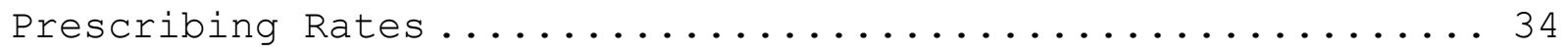

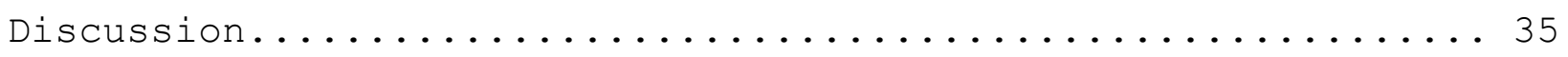

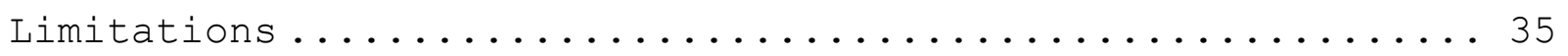

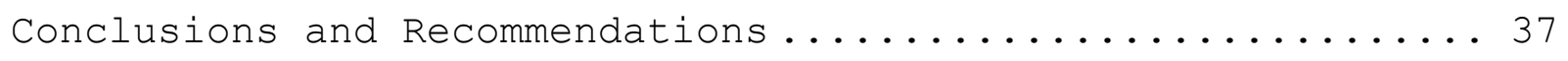

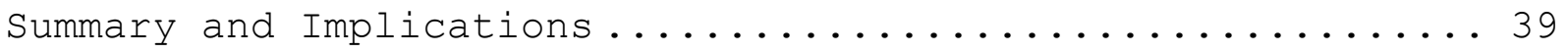

Attainment of Leadership Goals ................... 40

Appendix $A \ldots \ldots \ldots \ldots \ldots \ldots \ldots \ldots \ldots \ldots \ldots \ldots \ldots \ldots \ldots \ldots \ldots$

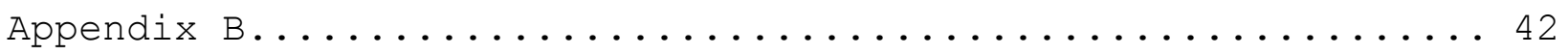

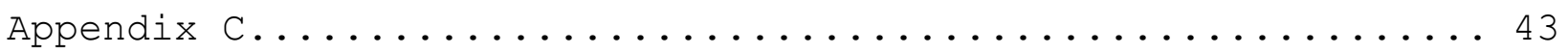




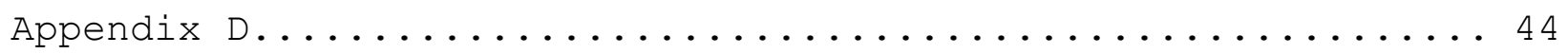

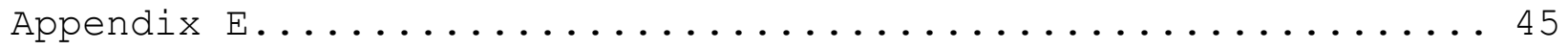

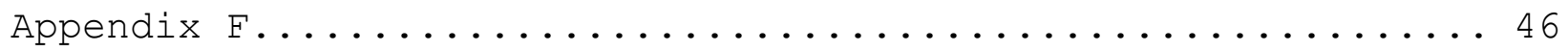

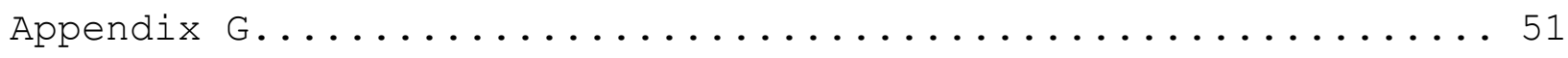

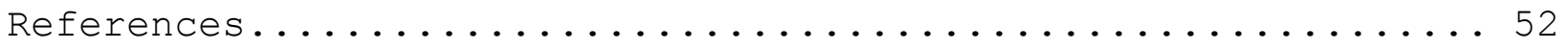




\section{Introduction}

Antibiotic use for hospitalized patients continues to increase, as do the complications, costs, and morbidities from diarrhea associated with antibiotics. Probiotics have been shown to prevent or reduce the incidence of diarrhea associated with antibiotics. This project investigated the effect of an education session and prescription guideline on the knowledge, attitudes, and beliefs of physicians and nurses regarding probiotics, as well as on rates of prescribing a probiotic concurrently with antibiotic therapy pre and post intervention.

\section{Background and Significance}

Over half of all hospitalized patients are treated with antibiotics, which accounts for 20-50\% of all hospital drug costs (Pestotnik, Classen, Evans, \& Burke, 1996). Even though there is concern about bacterial resistance, antibiotic use at United States hospitals is rising (Dunham, 2008). At a group of United States academic medical centers, antibiotic use rose 7 percent from 2002 to 2006 (Pakyz, MacDougall, Oinonen, \& Polk, 2008). Antibiotic associated diarrhea (AAD) is a frequent side effect of antibiotic use. In the last decade, there has been a 500\% increase in cases of AAD (Meier, 2005).

The clinical presentation of AAD ranges from mild to severe. Pseudo membranous Clostridium difficile (C. difficile) 
is a toxin-induced colitis and the most severe manifestation of AAD (Meier, 2005). In up to 70-80\% of diarrhea cases, the cause of the diarrhea is not specific, and in $20 \%$ it is due to $C$. difficile (Hurley \& Nguyen, 2002). In hospitalized patients, AAD has been associated with increases in mortality, length of stay, and cost of medical care (Brossard \& Surawicz, 2004).

One of the major mechanisms for the development of AAD is related to an alteration in the intestinal flora. The intestine is one of the largest bacterial reservoirs in humans (Gill \& Guarner, 2004). The organisms present in the intestine are delicately balanced to benefit both the organism and the host. A multitude of factors can disrupt this balance including food, drugs, general health, and alteration of the types and numbers of bacteria present (Gill \& Guarner). One of the most significant causes of disturbance of the gastrointestinal flora is antibiotic therapy (Sellin, 2001). The normal anaerobic gut microflora metabolizes high-molecular-weight carbohydrates into absorbable short-chain fatty acids. When the gut microflora is altered by antibiotics, high-molecular-weight carbohydrates accumulate in the colon and cause osmotic diarrhea (Reisinger, Fritzsche, Drause \& Drejs, 2005). Antibiotics that can reduce the number of gut anaerobes are aminopenicillins, cephalosporins, and clindamycin (Doron, et al., 2008); however, almost every antibiotic has the potential to cause AAD and $C$. 
difficile, including the antibiotics used to treat $C$. difficile (Hurley \& Nguyen, 2002).

Disturbances of the gut microflora also promote the overgrowth of distinct pathogenic bacteria. About 16-20\% of AAD cases are caused by C. difficile (Chassany, Michaux \& Bergmann, 2000, Reisinger et al., 2005, Sellin, 2001). The triggering event for $C$. difficile diarrhea is disruption of the normal colonic microflora, usually caused by a broad-spectrum antibiotic (Hurley \& Nguyeun, 2002). After the disruption of the colonic microflora, colonization of $C$. difficile occurs by ingestion of heat-resistant spores that convert to vegetative forms in the colon. The effect of an antibiotic on the intestinal system depends on the antibacterial spectrum and the concentration in the intestinal lumen (Chassany et al., 2000). Moreover, antibiotics with the broadest spectrums like penicillins, cephalosporins, and clindamycin, and those with a high intraluminal concentration in the intestinal tract lead to greater changes in the normal flora of the intestines (Chassany et al.). The more profound the alteration of intestinal flora by a given antibiotic, the more likely it is to cause AAD (Doron, Hibberd \& Gorbach. 2008). Bile acids, which escape absorption in the small bowel, are usually deconjugated and dehydroxylated by bacteria. When the bacterial flora is disturbed, unmetabolized dehydroxy bile acids, which are potent secretory agents, lead to 
development of secretory diarrhea in the colon (Reisinger et al., 2005).

Some antibiotics, such as erythromycin, display a molecular design similar to that of the paracrine peptide motilin, which induces intestinal motility. Thus, erythromycin may bind to and stimulate the motilin receptors, stimulating transit in the intestine, with resulting increase in intestinal contractility (Sellin, 2001). Clavulanate also stimulates small bowel motility.

Other contributing risk factors for the development of AAD are prolonged use of antibacterial therapy, repeated antibiotic therapy, and the combination of antibiotics. The highest incidence of AAD in hospitals is in the intensive care unit (Bergogne-Berezin, 2000). Additional risk factors for AAD include: patient immune status and age, route of antibiotic administration, and inpatient/outpatient status (Boyle, RobinsBrowne, \& Tang, 2006). It has been proposed that patients less than six years of age or greater than 65 years of age, with a past history of AAD, with severe underlying diseases, with chronic disease of the GI system, immunosuppression, GI surgery, or who are receiving antibiotics via nasogastric tube have increased risk of AAD (Bergogne-Berezin).

The rate of $C$. difficile acquisition is estimated to be 13\% in patients with hospital stays of up to two weeks and 50\% in 
those patients with hospital stays longer than four weeks (Schroeder, 2005), meaning that patients could experience this complication well after hospital discharge. C. difficile diarrhea can occur up to eight weeks after discontinuation of antibiotics (Hurley \& Nguyen, 2002). The cost of treating $C$ difficile infections is $\$ 2000-\$ 4000$ per hospital stay (Broussard \& Surawics, 2004; Hickson, 2007).

\section{Significance of Probiotics}

Probiotics are defined as live microorganisms that, when administered in adequate amounts, confer a beneficial effect on the health of the host (FAO/WHO, 2001). Probiotics modulate mucosal and systemic immunity, as well as improve nutritional and microbial balance in the intestinal tract (Penner, Fedorak, \& Madsen, 2005). Probiotics are known to: colonize the intestinal tract, repopulate the gut with nonpathogenic flora, enhance immune responses, and inhibit or even kill pathogenic bacteria (Doron, et al., 2008).

Probiotics are lactic acid producing cultures that stimulate colonization of the human original flora in a beneficial direction (Cedgard, 1998). A microorganism must exhibit certain criteria to be a probiotic. It must be of human origin, safe in nature, unaffected by gastric acid and bile, adhere to intestinal mucosa, and be able to produce antimicrobial substances, modulate immune responses, and 
influence human metabolic activities (Hanaway, 2006). Probiotic dosages are expressed in billions or millions of organisms, for example $10^{10} \mathrm{cfu}$ (colony forming units). Probiotics exhibit strain-specific differences in their resistance to acid and bile, ability to colonize the gastrointestinal tract, clinical efficacy, and benefits to the health of the host (Pham, Lemberg \& Day, 2008). Bifidobacteria and lactobacilli are commonly used as probiotics (Gill \& Guarner, 2004). Lactobacillus rhamnosus GG (LGG) and Saccharomyces boulardii have the most level 1 evidence to support use in preventing antibiotic associated diarrhea (Pham, et al.). Probiotics are a safe, cost effective measure to prevent or reduce AAD (Hickson, et al., 2007).

\section{Safety of Probiotics}

The safety of LGG has been evaluated more than any other probiotic (Snydam, 2008). In the United States, most probiotics have the status of generally recognized as safe (GRAS), therefore probiotics are not subject to specific standards (Vanderhoff \& Young, 2008). However, LGG has been rigorously studied in academic institutions. These studies have validated the effectiveness of LGG with AAD (Vanderhoff \& Young). LGG has shown to be safely used in many human populations including pregnant women, premature neonates, the elderly, children with diarrhea from rotovirus, hospitalized children and adults with diarrhea, malnourished children, patients with rheumatoid 
arthritis, adults with Crohn's disease, adults with Helicobacter pylori infection, and adults with $C$. difficile diarrhea (Snydman). There have been rare cases of bacteremia and liver abscess in patients with short gut syndrome (Snydam). All probiotics should be used with caution in patients who: are immune compromised, have short bowel syndrome, central venous catheters, elderly patients, and those with cardiac valve disease (Syndam). In the institution where the practice change was undertaken, patients are considered immune compromised if they are: receiving chemotherapy for cancer, have bone marrow suppression, are hemodynamically unstable beyond a few days or have multiple organ failure, have received an organ transplant, have a significant history of alcohol abuse or have a white blood cell count of $<4000$ or absolute polymorphonuclear leukocyte <1000 (H. Dedhia, personal communication, April 15, 2009). Since probiotics are viable organisms, it is feasible that the host could become infected by them. However historical data shows that lactobacilli in either food or capsule form are safe for human use (Reid, 2003).

It is estimated that there are more than 20 billion doses of probiotics taken in a year and only a few reports of bacteremia (Reid, 2005). In a retrospective study by Salminen, et al. (2004), there were 89 cases of lactobacilli bacteremia, of which 11 might have been related to probiotic LGG use. In 82\% 
of these cases, patients had severe or fatal comorbidities. In Finland, the annual per capita consumption of LGG increased from 1 liter to 6 liters between 1995 and 2000. Researchers in Finland studied all Lactobacillus blood isolates from 1990-2000 and found that the rate of Lactobacillus bacteremia remained constant over time (Ouwehand, Saxelin, \& Salminen, 2004 \& Salminen, et al.). Even though probiotic use appears to carry a very low risk, Doron, et al. (2008) recommends that healthcare providers change gloves and wash hands after handling LGG and before manipulating vascular catheters.

\section{Probiotic Dose}

According to the World Gastroenterology Organization (2008) and Hickson, et al. (2007), the recommended dose of LGG for prevention of $A A D$ in adults is $10^{10}-10^{11}$ cfu, BID. This dose was based on evidence from well-designed and properly powered clinical trials.

\section{Problem Statement}

A large majority of patients admitted to the medical intensive care unit at the selected trauma center receive antibiotic therapy, with many receiving multiple antibiotics concurrently, putting them at high risk for AAD. However, few patients are prescribed probiotic therapy concurrent with the 
antibiotic treatment, an intervention that might significantly reduce the risk of AAD.

\section{Theoretical Framework}

Two theories guided this capstone project, one serving as a framework for the patient intervention, and the other a framework for the provider intervention.

\section{Homeostasis Theory}

Claude Bernard's Homeostasis Theory framed the patient intervention. In the mid $1800^{\prime}$ s Bernard described the importance of the concept of the constant internal environment. Bernard believed that life is an expression of the physical reality and the maintenance of life is guaranteed by the constancy of the fluid matrix or milieux interieur (Bernard. 1927). Cannon coined the term homeostasis to describe the constancy of the internal variable and the regulatory integrated mechanisms directed to preserve it (Cannon, 1929). Homeostasis does not mean the environment is immobile or stagnate, but rather that conditions vary, but remain relatively constant (Cannon). Shortly after birth, humans become colonized with microbes that are dynamic components of the body (CAST, 2007). Intestinal microbes are fairly stable through the life span, but can be impacted by antibiotics (CAST). Homeostasis is about the condition of an optimal internal environment for cell and tissue function at any 
moment in time. Health occurs when bodily function is able to provide the appropriate environment (McVicar \& Clancy, 1998). Failure to provide an optimal internal environment will cause further destabilization, and the integration of physiological functioning will become impaired. A change in the activities of one system may have far-reaching consequences for whole body function (McVicar \& Clancy).

The human gastrointestinal tract is home to over 400 microorganisms. Most of this indigenous flora exhibit health promoting properties, but some can cause disease. Usually there is a balance between the good and bad bacteria. However, the use of antibiotics is known to exert a significant influence on the number and species of microorganism that inhabit the gut (Gill \& Guarner, 2004). Probiotics can help restore the body's normal intestinal flora and the internal environment, thereby maintaining homeostasis and decrease the risk for AAD and C.difficle, as they occur due to a disruption in the homeostasis of the GI tract.

\section{Change Theory}

Lewin's Change Theory (Lewin, 1997) was used to guide the practice change. Lewin's theory consists of three essential stages: unfreezing, moving to a new level or changing, and refreezing. Unfreezing involves a method of making it possible for health care providers to change their behavior. To 
facilitate unfreezing education was provided to both physicians and nurses.

When thoughts, perceptions, feelings, and attitudes move to a new level, a change has occurred. A guideline was provided to assist physicians in the process of ordering probiotics. Additionally, the project director participated in daily rounds for the first two weeks of the project.

Refreezing is establishing the change as a new habit. This would occur when it becomes the standard of practice to order probiotics with certain antibiotics. If refreezing does not occur, the old behavior returns (Schein, 1995). This would mean that probiotics would not be ordered.

According to Schein (1995), Lewin's concept of unfreezing emphasizes the observation that the stability of human behavior is based on "quasi-stationary equilibria" supported by a large force field. Lewin assumes that in any situation there are both driving and restraining forces that influence any change that may occur. These forces can be positive, influencing one toward a behavior, or negative, pushing one away from a behavior. Driving forces are those forces affecting a situation that are pushing in a particular direction. Driving forces tend to initiate a change and keep it going. However, restraining forces are forces acting to restrain or decrease the driving forces (Schein). 
For this project unfreezing activities included the education program which contained information regarding antibiotic associated diarrhea and the benefit of probiotic use. Additionally an evidence-based guideline was developed. The education was presented to the physicians who order probiotics and to the bedside nurse, who could facilitate the ordering of the probiotic. During the moving phase, the project director maintained close contact by participating in rounds with the physicians in the MICU. This allowed for questions to be answered and encouragement to be given for the practice change. The evidence-based practice guideline was in place to assist new residents who rotate through the unit as well as other physicians. Driving forces included: encouragement from the project facilitator, pressure from the staff physicians, reminders from the bedside nurse, and enthusiasm or "buy in" generated from the educational program. Restraining forces included health care providers that doubt the benefit of probiotics and hence do not order them. Theoretically, in the refreezing phase, physicians in the MICU would be consistently ordering probiotics for patients receiving antibiotic therapy and nurses would support this practice. The effectiveness of the change was monitored by comparing prescribing rates per and post guideline introduction and education. 


\section{Project Description}

\section{Literature Review}

In order to identify potential practice protocols, a thorough literature search was conducted. Data bases searched included the National Guidelines Clearinghouse, the Cochrane Library, Pub Med, and CINAHL from 1999 to present. Search terms were probiotics, antibiotics, adults, and diarrhea in various combinations. The National Guidelines Clearinghouse yielded one guideline but it was not specific to probiotics and diarrhea. The Cochrane Library yielded three hits: two were protocols not yet developed and one was a review on probiotics for the prevention of pediatric antibiotic-associated diarrhea. Pub Med initially yielded 2201 hits, with probiotic as the search term. Adding diarrhea narrowed the search down to 545, which was further narrowed to 40 by adding antibiotics and adult. An exhaustive review was completed on this search and 7 articles were retrieved for this paper. Through the use of snowballing the article from the WHO organization was obtained.

Seven articles will be reviewed, three randomized control trials, one systematic review, and three meta-analyses. All of the studies address the use of probiotics with AAD and were published from 2002-2007.

The focus of a study by Cremonini, Di Caro, Nista, Bartolozzi, Capelli, G. Gasbarrini, et al. (2002) was to perform 
a meta-analysis of published trials on the efficacy of probiotics in reducing the incidence of AAD. Inclusion criteria were: only placebo-controlled trials, diarrhea as the primary end-point, and only studies with a minimum of two week followup. Data regarding diarrhea was based on presence or absence of diarrhea; results based on differences in the amount of daily stool were excluded from analysis. Only seven placebo-controlled trials matched the inclusion criteria. The trials included used Lactobacillus spp. or Saccharomyces boulardii a single probiotic species instead of a combination of probiotics. A total number of 881 patients were studied in the trials, with an age range of two weeks to elderly. Of the seven trials reviewed, three identified a decrease in the occurrence of AAD during administration of Saccharomyces boulardii and four during the administration of Lactobacillus spp. The results of this study showed an overall reduction in the risk of AAD during probiotic administration in the studies considered. It was further noted that even though data suggests a positive role for probiotics in AAD, lack of standardization of probiotic preparations may impact research findings. The author calls for more equivalent probiotic formulations.

D'Souza, Rajkimar, Cooke and Bulpitt (2002) performed another meta-analysis to evaluate the efficacy of probiotics in prevention and treatment of diarrhea associated with the use of 
antibiotics. All randomized, double blind trials that compared the effects of probiotic therapy and placebo were included. The percentage of patients without diarrhea in the probiotic and placebo groups was used as the outcome measure. Nine trials were included in the final analysis. All trials studied the efficacy of a probiotic in the prevention of AAD. The studies used probiotics combined with one or more antibiotics. The numbers of patients and duration of follow-up varied greatly from study to study, but the patients' characteristics were similar in both treatment and placebo group. The combined odds ratios were similar between the yeast trials (Saccharomyces boulardii) and the non yeast trials; both favored active treatment over placebo in the prevention of AAD. The odds ratio for pooled data from all nine trials was in favor of probiotics over placebo in the prevention of AAD $0.37(0.26$ to $0.53 ; \mathrm{P}<0.001)$. Six studies showed a significant benefit of probiotic treatment compared with placebo. This meta-analysis concluded that probiotics may be useful in preventing $A A D$, but it provided little support for treating AAD already in existence. Moreover, it indicated that probiotics are increasing in availability, have a low cost, and lack side effects in contrast to the problems associated with antibiotics.

In a randomized trial, Beniwal, Arena, Thomas, Narla, Imperiale, Chaudhry, et al. (2003) studied the effectiveness of 
a dietary supplement of yogurt for the prevention of AAD. Two hundred two hospitalized adult patients receiving oral or intravenous antibiotics were randomized to receive or not to receive a dietary yogurt supplement. The yogurt contained $10^{6}$ cultures of L. acidophilus, L. bulgaricus, and S. thermophilus combined. The intervention group received yogurt twice daily for eight days within 12 hours of starting an antibiotic. Within each block, six subjects were randomized to one of two groups to ensure a balance in the number of subjects assigned to receive yogurt or no treatment. Randomization was stratified according to whether patients were receiving $C$. difficile treating antibiotics, metronidazole or vancomycin, or non $C$. difficile treating antibiotics. Treatment assignment was not revealed to the patient until they agreed to enroll in the study. Yogurt decreased the risk of developing AAD by nearly 50\% ( $p=0.04)$. The total number of diarrheal days was 60 in the control group and 23 in the yogurt group. The incidence of AAD in the control was $23.7 \%$ which is consistent with other reports of frequency of AAD. The incidence of AAD in the yogurt group was $12.4 \%$. Based on the results, the absolute risk reduction implies that nine patients (95\% confidence interval 4.1-132.6) need to be treated with yogurt to prevent one case of AAD. The study was not double-blinded due to the nature of the intervention. However, patient responses were elicited in a standardized fashion by 
certified dieticians, which may have mitigated the effect of the lack of blinding. Incorporating a treatment arm consisting of yogurt without active cultures would have strengthened the study. It is also noted that the results are based on the combination of specific probiotics; therefore, the results cannot be applied to single probiotics or other dosages or combinations. This study demonstrated that during the course of antibiotic therapy, supplementation with commercially available yogurt that contains active cultures is a simple, safe, and cost-effective method of reducing the occurrence and severity of AAD .

Hawrelak, Whitten, and Myers (2005) performed a systematic review to determine if co-administration of LGG with antibiotics reduced the subsequent incidence of AAD. A systematic review was done on six, placebo controlled trials that compared the effects of a probiotic and a placebo on AAD. Statistical heterogeneity of the trials did not allow meta-analysis. The heterogeneity of the systematic review was found to be due to one study; without the one study, the trial results were all statistically homogenous. Four of the six trials found a significant reduction in the risk of AAD with co-administration of LGG. One of the trials reduced the number of days with AAD and the final trial found no benefit. All patients in the four positive trials received oral antibiotics and were outpatients. The participants 
in the negative study were inpatients and an unspecified number of patients received IV antibiotics. There were a total of 638 participants, ages two weeks to 93 years. Daily doses of LGG ranged from $1 \times 10^{10}$ to $4 \times 10^{10}$. Duration of treatment days ranged from 7-14 days. Limitations of this systematic review include a small number of studies that examined the use of LGG and AAD. Some of the studies had a small number of participants. Additionally different antibiotics were used, as well as different dosages of LGG. LGG is the probiotic to be administered in this project and most of the studies showed a decrease in the percentage of subjects with diarrhea: 5\% vs 16\%, $8 \% \operatorname{Vs} 26 \%, 29 \% \operatorname{Vs} 30 \%, 3 \% \operatorname{Vs} 27 \%$ and $5 \% \operatorname{Vs} 30 \%$

McFarland (2006) performed a meta-analysis to compare the efficacy of probiotics for the prevention of AAD and the treatment of $C$. difficile disease (CDD) based on published randomized, controlled clinical trial. Twenty-five randomized controlled trials, with a sum total of 2,810 patients, provided data regarding efficacy of probiotics. The types of probiotics varied from single strains to mixtures of probiotics. Daily doses ranged from $1 \times 10^{7}$ to $1 \times 10^{11}$. Use of a high dose $(>=$ $10^{10} /$ day) of probiotic was associated with more effectiveness with AAD. The duration of probiotic treatment also varied from five days to eight weeks. In this meta-analysis, three types of probiotics (Saccharomyces boulardii, LGG, and probiotic 
mixtures) significantly reduced the development of AAD. Only Saccharomyces boulardii showed significant reductions in recurrences of CDD. Limitations of this study include the varying types of probiotics and antibiotics, varying doses, varying age of participants, and variations in sample size. Although there were limitations, the data synthesis from twentyfive randomized controlled trials showed that probiotics significantly reduced the relative risk of AAD. From six of the randomized controlled trials the combined efficacy showed probiotics have a significant protective effect for CDD.

A randomized, double blind, placebo controlled trial by Hickson, D'Souza, Muthu, Rogers, Want, Rajdumar, et al. (2007) studied the efficacy of a probiotic drink containing lactobacillus for the prevention of diarrhea associated with antibiotic use and diarrhea caused by $C$. difficile. One hundred and thirty five hospitalized patients taking antibiotics were studied. The treatment group received a probiotic drink containing $L$ casei imunitass, (1.0 x $\left.10^{8} \mathrm{cfu} / \mathrm{ml}\right), S$ thermophilus $\left(1.0 \times 10^{8} \mathrm{cfu} / \mathrm{ml}\right)$, and L. bulgaricus $\left(1.0 \times 10^{7} \mathrm{cfu} / \mathrm{ml}\right)$. The placebo group received a sterile milkshake. The treatment group and placebo group received the drink twice daily during antibiotic treatment and for one week after the antibiotic was completed. Twelve percent of the probiotic group developed AAD compared to $34 \%$ in the placebo group. This study concluded that 
consumption of a probiotic drink containing L casei, L bulgaricus, and $S$ thermophilus can reduce the incidence of AAD. The study could not establish which bacteria species was effective. It is possible that the bacteria work synergistically, or one species may be more effective than another. As a result, the results cannot be generalized to other probiotic products.

In the most recent placebo-controlled, double blind study by Koning, Jonkers, Stobberingh, Mulder, Rombouts, and Stockbrugger (2007), the effect of a multispecies probiotic on the composition and metabolic activity of intestinal microbiota and bowel habits was studied in healthy volunteers taking amoxicilin. Forty subjects were enrolled in the study, 19 in the probiotic group and 21 in the placebo group. Volunteers received $500 \mathrm{mg}$ of amoxicillin twice daily from day $1-7$ and were randomized to receive either 5 grams of a multispecies probiotic or 5 grams of a placebo twice daily from day 1-14. The probiotic contained 10 different bacterial species at each $10^{8} \mathrm{cfu} / \mathrm{g}$, the total does being $10^{9} \mathrm{cfu} / \mathrm{g}$ (b. bifidum, B longum, E. faecium, L. acidophilus, I. paracasei, la. Plantarum, LGG, and 1. salivarius. Congruent with other studies, the intake of a multispecies probiotic resulted in a decrease in diarrhea-like bowel movements. During the probiotic period, diarrhea was reported less frequently in the probiotic group (48\%) than in 
the placebo group (79\%). The study used healthy adult volunteers who received the same oral antibiotic and either a multispecies probiotic or a placebo.

\section{Synthesis}

For the purpose of this literature review, three randomized controls, one systematic review, and three meta-analyses were examined. In all of the studies reviewed from 2002-2007, patients receiving a probiotic reported less frequent diarrhea. Cremonini, et al. (2002) showed an overall reduction in the risk of AAD during probiotic administration. D'Souza, et al. (2002) concluded that probiotics may be useful in preventing AAD, but provided little support for treating AAD. Beniwal, et al. (2003) showed that during the course of antibiotic therapy, supplementation with commercially available yogurt that contains active cultures is a simple, safe, and cost-effective method of reducing the occurrence and severity of AAD. Additionally, Hawrelak found that when LGG was administered, there was a decrease in the percentage of subjects with diarrhea. In McFarland's meta-analysis, the data synthesis from twenty-five, randomized, controlled trials showed that probiotics significantly reduced the relative risk of AAD. Hickson, et al. (2007) and Koning, et al. (2007) also showed a decrease in the incidence of developing AAD when the patient was given a probiotic. 
Based on a review of the literature, there is sufficient evidence to support the practice of administering probiotics concurrently with antibiotics in high risk patient populations, after screening for potential contraindications. The proposed practice change project is supported by evidence found in the literature.

\section{Congruence of Organizations Strategic Plan}

This project supports the mission, values, goals, and strategic plan of West Virginia University Hospital (WVUH). The mission of WVUH is to provide a quality healthcare system, including tertiary services, to the citizens of West Virginia and the surrounding region (WVUH, 2008). WVUH (2008) values patient, team, and community. The mission of WVUH is achieved through strategic planning with a focus on improving the health status of the local community and the state of West Virginia and maintaining a strong financial position (WVUH). This project was designed to assist in achieving the mission by improving the health status of the patients in MICU. The use of probiotics can promote returning of the patients' intestinal microflora to a pre-antibiotic state, thus potentially decreasing mortality and morbidity (Hickson, et al. 2007). Based upon the findings of a study by Hickson, et al., it is estimated that a decrease in patient length of stay might also occur. 


\section{Project Objectives}

The main objective of the project was to introduce a guideline for ordering probiotics concurrently with antibiotics. An additional objective of the project was to increase knowledge of physicians and nurses regarding incidence and effect of AAD and the use of probiotics to reduce the risk for and length of AAD. Another objective was to achieve a 50\% increase in prescribing rates of probiotics for patients receiving aminopenicillin, cephalosporin, and clindamycin type antibiotics in the medical intensive care unit at a Midatlantic academic hospital by July 2009. The probiotic was to be ordered concurrently with the antibiotic by the ordering physician. An additional objective was to impact physician and nurse attitudes about the safety and effectiveness of probiotic use by presenting an educational program about the benefit of probiotic use with antibiotics in patient care. The project was completed during the period of May-July 2009 (Appendix G) .

\section{Project Design}

\section{Evidenced Based Intervention Plan}

A Curriculum for the Role of Probiotics in the Prevention of Antibiotic Associated Diarrhea was developed (Appendix A). Additionally, an evidence-based practice guideline for prescribing probiotics was developed to assist physicians and 
nurses in ordering probiotics concurrently with identified antibiotics (Appendix E). A pretest/posttest was developed based on a review of the literature. The assessment test was reviewed for clarity and readability by ten nursing faculty at the school of Nursing prior to administering the test.

The educational program about probiotics and AAD as well as the guideline for ordering probiotics was presented at a research rounds to physicians in the MICU. The nurses in the MICU were educated by through use of a poster presentation. An informational poster was placed in the break room in MICU 1, MICU 2, and SICU. Placing posters in all 3 units assured easy access to the nurses.

Prior to the education, a Probiotic Knowledge, Attitude, and Belief Assessment pretest was placed in the mailbox of all nurses in the MICU and SICU (Appendix F). In addition a letter explaining the project and thanking the nurses for their participation was placed in each mailbox. The education program was approved for continuing education credit; thus a contact hour was given to all nurses who completed the pretest and posttest. The pretests were collected throughout a 2 week period and then the posters were placed in each unit. The poster remained in the units for 6 weeks, with posttests being collected periodically throughout the 6 weeks. Certificates for 
contact hours were given to all nurses who completed the postest.

The pretest/posttest is a quasi-experimental design as it measures change within groups. Additionally, prescribing rate of probiotics for six weeks prior to and six weeks post intervention and for 13 weeks after the project ended were obtained from a data analyst for the hospital. The number of patients admitted to the unit, the number of antibiotics prescribed, and the numbers of c difficile diagnoses were also collected.

\section{Resources}

The resources for the project included the time of the MICU director, Dr. Dedhia, and other MICU physicians, residents, bedside nurses, MICU nursing administrators, pharmacists, and dieticians. The room and equipment that is normally used for grand rounds was used for the educational program. The budget included a cost of $\$ 37.00$ for preparing the posters. Education for physicians occurred during research rounds, a routine education time. The nurses' education did not take nurses outside of the care setting, as it occurred via poster board format. Nurse champions were informally identified during the education process through expressed interest and enthusiasm for the project. This allowed for promotion of the project during times when the project director was not on the unit. Probiotics 
are currently covered by insurance, thus there is generally no cost to the patient receiving them. The cost of LGG is $\$ 0.14$ per dose (G. Gill, personal communication, September 22, 2008) and insurance is billed the minimum charge of $\$ 3.26$ (G. Gill personal communication, September 24, 2008).

\section{Evidence of Key Site Support}

Harakh V. Dedhia, MD, medical director of the medical intensive care unit was in support of the project (Appendix B). Dr. Dedhia is board certified in internal medicine, pulmonary disease, and critical care medicine, with a special interest in nutrition. Chris Frenecak, a registered dietitian for MICU, was also in support of the project (Appendix C). Mary Fanning, Nursing Administration Director, granted permission to complete the project in the MICU (Appendix D).

\section{Evaluation}

The project was evaluated in two ways. The health care provider's knowledge, attitudes, and beliefs toward probiotic use were evaluated and probiotic use was monitored. A pretest was given to the health care providers before the education program and a posttest followed the education program. Probiotic prescribing rates, MICU admissions, antibiotic prescribing rates, and $C$. difficile rates were obtained from the data analyst for six weeks prior to the education program and 
for six weeks after the intervention, as well as for 13 weeks after the project ended.

A paired t-test was used to compare knowledge scores from the pretest and posttest data. Pre and post intervention prescribing rates were compared to determine if the goal of a $50 \%$ increase was met. In addition antibiotic prescribing rates, MICU admissions, and $C$. difficile rates were also compared.

\section{Results}

\section{Demographics}

Seventy-one percent of the 45 participants completing the pretest were registered nurses, 16\% were physicians, 11\% were dieticians and 2\% were respiratory therapists. Forty-two participants completed the posttest: $64 \%$ were registered nurses, 18\% were physicians and 11\% were dietitians.

Average length of time in practice for all health care providers was 11.7 years. Physicians had an average of 16 years with a range of 1-35 years of practice. Years of experience for nurses ranged from 0-33 years, with an average of 9.34 years. Dietitians had an average of 21 years of experience, with a range of $12-35$ years. One respiratory therapist completed the testing with 8 years of experience. 


\section{Pretest/Posttest Results}

The survey measured knowledge, attitude, and beliefs. A change in knowledge from pretest to posttest was measured with a paired t test by computing total scores for knowledge on 25 items. The paired t test indicated a significant increase in test scores after education, $t(d f=44)=7.192, p=.000$. Mean knowledge scores increased from $11.1(\mathrm{SD}=2.68)$ pretest to 18.2 $(S D=6.22)$ posttest. 


\section{Table 1: Knowledge Items: Percent Correct Pre and Post Test}

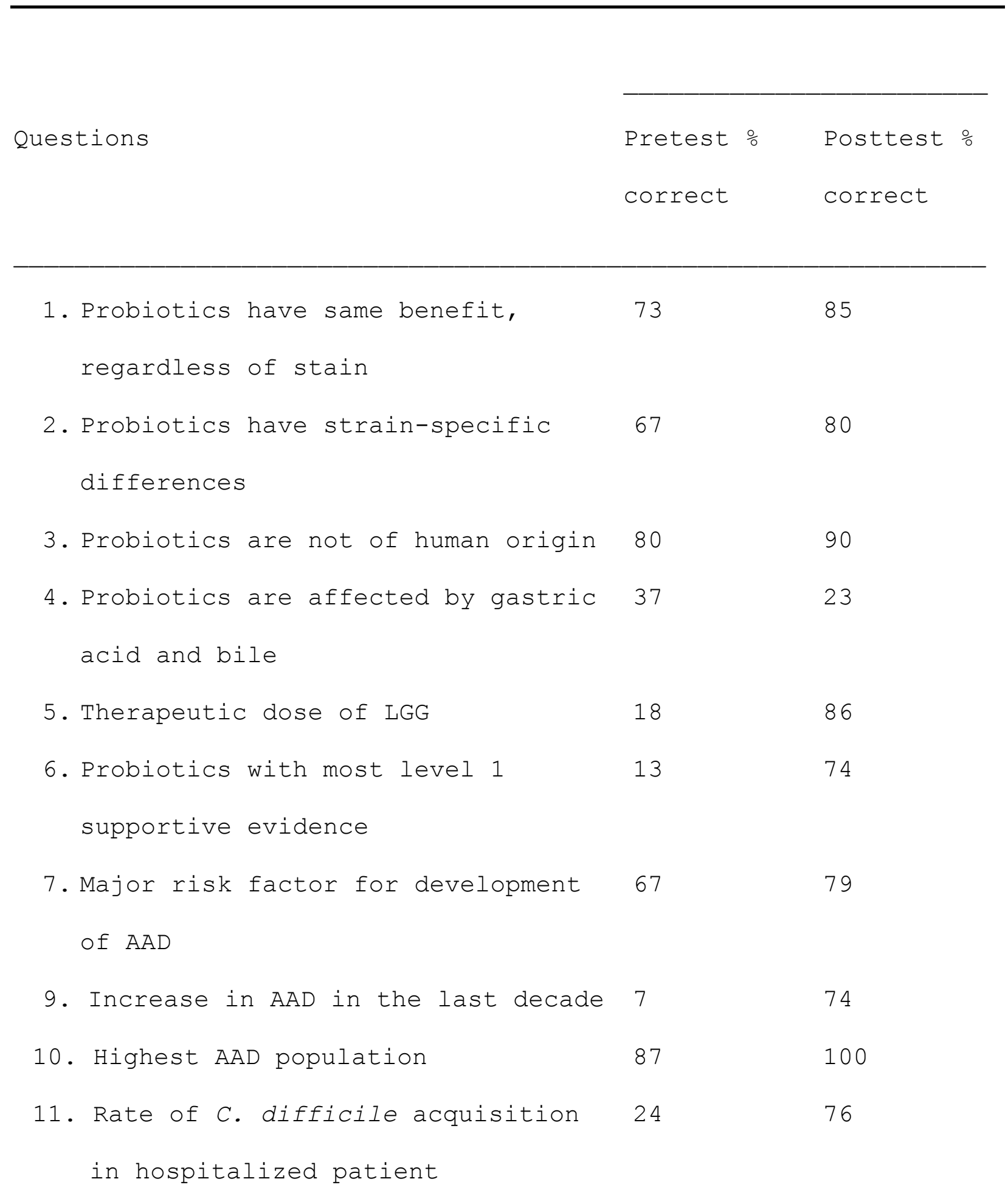


Questions

12. True/false all antibiotics cause
Pretest $\%$ Postest $\%$

correct correct

AAD

Name three classifications of antibiotics that reduce gut anaerobes.

13. One

14. Two

15. Three

Probiotics should be used with caution

in patients who

16. Are immune compromised,

65

95

17. Are receiving multiple antibiotics,

18. Have short bowel syndrome,

61

88

19. Have a central venous catheter,

20. Are elderly,

67

78

21. Have cardiac valve disease.

88

Precaution when handling a probiotic

22. No precaution, 
Questions handling,

24. Use sterile gloves,

25. Change clean gloves and wash hands before central catheter
Pretest $\frac{\circ}{0} \quad$ Posttest $\frac{0}{0}$

correct correct

19

7

100

95

49

85 
Some knowledge questions changed substantially from the pretest to the posttest. Only 18\% correctly identified the therapeutic dose of LGG on the pretest, while 86\% were able to identify the correct dose on the posttest. Similarly, only $4.5 \%$ correctly identified the rise in antibiotic use from 2002 to 2006 on the pretest, whereas 79\% answered correctly on the posttest. In addition, 7\% correctly identified the increase in AAD over the last decade on the pretest, and $74 \%$ on the posttest. Only $24 \%$ were able to identify the incidence of $C$. difficile acquisition on the pretest, whereas 76\% were able to identify the incidence on posttest. Around 30\% of participants were able to name three classifications of antibiotics that reduced the number of gut anaerobes on the pretest, whereas over 90\% were able to name three classifications of antibiotics on the posttest. Lastly, the greatest knowledge change regarding caution in probiotic use was identification of caution in patients who have a central venous catheter. On the pretest $12 \%$ answered this item correctly, and on the posttest 78\% answered correctly.

Attitude change was measured by computing total score for items indicating the level of agreement/disagreement with statements about the prevalence and severity of AAD as a health problem and the usefulness, safety and effectiveness of probiotic use concurrently with antibiotics. Low scores 
represented the most positive attitude. There was a significant difference in pre and post scores, $t(d f=41)=2.86, p=.007$. Mean attitude scores decreased from 12.3 to 10.6, indicating a more positive attitude toward the significance of AAD as a health issue and the potential benefit of probiotic use. After education, the reported likelihood of prescribing probiotics together with antibiotics also increased significantly, $t$ (df= $38)=3.13, p=.003$. Mean scores decreased from 2.7 to 2.0.from pretest to posttest.

Only 19 (42\%) participants had prescribed or recommended a probiotic and 25 (56\%) had never prescribed or recommended a probiotic. Twenty-nine (64\%) of participants have known people who have used probiotics and 16 (36\%) did not know of anyone who used probiotics. Twenty-five (56\%) had personally used probiotics either in food sources or supplements and 20 (44\%) had not used a probiotic.

Themes in the open response items for reservations about recommending or prescribing probiotics included not being well informed on probiotic use and not having seen probiotics used in practice. Themes identified with the question, "What might encourage you to prescribe or recommend probiotics?" included: more evidence of benefits, more education, increased knowledge, visual reminders, and ease of administration. 


\section{Prescribing Rates}

There were 148 patients admitted to MICU service 6 weeks prior to the education intervention, and of those patients, 96 (66\%) were prescribed antibiotics. Of the patients receiving antibiotics, 2 (2\%) received the probiotic LGG. There were 138 patients admitted to MICU service during the 6 week post education study period and of those, 82 (59\%) were prescribed antibiotics. Of these patients, 4 (5\%) were prescribed the probiotic LGG. There was a 2 fold increase in probiotic prescribing rates; however there were a very small number of probiotics prescribed in total. In the time frame post study period (June 25-August 31), there were an additional 2 patients prescribed the probiotic LGG. Hospital-wide, there were 106 patients prescribed a probiotic from January 17- September 18 , 2009. The overall probiotic prescribing rate was low throughout the hospital during this time frame. Probiotics received via a food source such as yogurt could not be determined, as this data is not tracked through the medical record system.

One of the two patients that received a probiotic before the education session had a confirmed diagnosis of $C$. difficile. Two of the four patients that received a probiotic during the six week post education intervention study period had a confirmed diagnosis of $C$. difficile, and another had diarrhea identified in the medical record. The two patients that received 
a probiotic during the post study period both had a confirmed diagnosis of $C$. difficile. Eleven patients listed under MICU services had documented $C$. difficile pre invention and 5 patients had documented $C$. difficile post intervention.

\section{Discussion}

\section{Limitations}

The purpose of this project was to initiate a practice change to increase probiotic use concurrent with antibiotic use in the MICU. Several issues may have impacted the effect of the practice change project. First, the project director was not present in the unit on a daily basis, to serve as a constant change agent. Even though the residents who were assigned to this patient population were provided education regarding the practice change, the residents in the unit change on a monthly basis. Thus, residents who prescribed antibiotics during the latter weeks of the post intervention period most likely had not attended the education intervention session. The staff physicians were also provided the education session; however the staff physician rotates weekly, and all staff MD's do not attend the research conference at which the education session was provided. The physician education session could not be conducted during grand rounds, where staff physician attendance might have been higher, due to scheduling conflicts. 
Another limitation was the low rate of nurse completion of the poster education session. Posters were available in multiple locations, to assure availability to all staff RNs, yet only 30 nurses (25\%) completed the pre and post test. Thus, a large number of $\mathrm{RN}^{\prime} \mathrm{s}$ may have been unaware of the proposed practice change on the unit. In addition, although the nurses on the unit can suggest and/or remind physician to order a probiotic, they cannot themselves prescribe a probiotic for a patient. It was not possible to collect data on nurse prompting behaviors; thus the potential impact of these behaviors is not known.

Another limitation that became apparent related to the different roles of the project director and the physician providers. The practice change was being proposed by a nurse practitioner to physicians in the MICU. Physicians may be reluctant to change practice based on a recommendation from a nurse practitioner.

In addition, an unexpected event occurred during the education session that may have impacted physician attitudes and behaviors related to the prescribing of probiotics. A pharmacist who attended the physician education session verbalized strong lack of support for the practice change. It appeared that he may not have been aware of the literature documenting the positive effects of probiotic use concurrent with antibiotics, but, nevertheless, voiced his concerns about the proposed practice 
change. His comments may have negatively influenced the attitudes and behaviors of the MDs and residents in attendance at the session.

Finally, it may be that the MICU is not the best unit to initiate the practice change of prescribing probiotics concurrently with antibiotics. The MICU patient population may have a higher prevalence of conditions for which probiotics use would be contraindicated or cautioned, such as immunocompromise, cardiac valve disease, and short bowel syndrome.

\section{Conclusions and Recommendations}

This project met the identified objectives to increase the knowledge of MICU physicians and nurses about the significance of $\mathrm{AAD}$ and the benefits of probiotics in reducing patient risk for this treatment complication, and to increase prescribing rates for probiotics concurrent with antibiotics in the MICU patient population. However, the impact on achieving the mission and strategic plan of the University Hospital was minimal at best, because the probiotic prescribing rate, while increased, remained very low.

As a result of this practice change project, several recommendations can be made for further interventions to promote the behavior of prescription of probiotics as adjuncts to antibiotic prescriptions. First, it might be best to attempt this practice change on a unit with both stable staff and stable 
prescribers. Then, education would be most likely to affect a change in practice. A second recommendation would be to implement the practice change on a unit with a non ICU population, where there would be less contraindications or cautions for use with the patient population.

An additional recommendation might be to use a yogurt containing LGG as an option for implementation of the probiotic, as physicians may be more willing to suggest yogurt for their patients than to prescribe a tablet that comes from the pharmacy. Use of yogurt might be perceived as "safer" than prescription of a formulary probiotic.

An additional recommendation would be to solicit champions from medicine, nursing, pharmacy, and dietary to promote the use of probiotics concurrently with antibiotic therapy. When there are champions in each area, the staff in those areas are more likely to participate in the practice change.

The use of a prompt or flag within the medication ordering system to remind providers to order a probiotic when ordering an antibiotic would, most likely, have a more significant impact on physician prescribing behavior. However, this change would require hospital-wide change in policy/procedure, and so would be a longer-term intervention. 
Finally, conducting research at the institution that may demonstrate the effectiveness of probiotics in preventing and/or treating diarrhea resulting from antibiotic therapy might increase credibility of this behavior and result in increased "buy in" by physicians. It would be possible to continue to collect data related to $C$. difficile rates, the cost of diarrhea and $C$. difficile in terms of hospitalization, and probiotic prescription rates. Perhaps as providers recognize more fully the effect of preventing even one case of $C$. difficile, they may be more amenable to taking the additional steps of prescribing a probiotic along with the antibiotics that are prescribed.

\section{Summary and Implications}

The education session did increase both physician and nurse knowledge of probiotics, and positively impacted their attitudes toward the use of probiotics in preventing AAD. In addition, the rate of probiotic prescription increased two-fold in the posteducation period, even though the prescription rate remained quite low. There were several intervening or hindering variables, which may have prevented a further change in attitude toward probiotics and probiotic prescribing rates. Given these intervening or hindering influences, several recommendations for future work in this practice change are suggested. 


\section{Attainment of Leadership Goals}

This project has contributed to my personal leadership goals in numerous ways. After coming up with a question, I was able to perform a literature review and systematically review research articles on the topic of probiotics and antibiotic associated diarrhea. I then incorporated two theories to guide the practice change. The practice change was designed to improve patient outcomes by applying research. I participated interprofessionally with physicians and nurses in the MICU to increase knowledge, change attitudes and beliefs, and increase prescribing rates of probiotics concurrently with antibiotics. I developed, implemented, and evaluated a practice change. I hope to continue to participate in promoting the use of probiotics concurrently with antibiotic therapy to prevent or decrease AAD in other areas of health care. 


\title{
Appendix A
}

\author{
Curriculum Outline \\ The Role of Probiotics in the Prevention of Antibiotic \\ Associated Diarrhea
}

1. What are probiotics?

2. The history of probiotics

a. Myths

3. Antibiotic associated diarrhea
a. The role of probiotics
b. Literature review
c. Recommendations
d. Safety Issues

4. Practice Change
a. When to order probiotics
b. When not to order probiotics
c. Dose 


\section{Appendix B}

\section{WestVurginiaUniversity}

SCHOOL OF MEDICINE

January 20, 2009

To Whom It May Concern:

RE: Probiotic Research Project at WVUH

I have reviewed the proposal by Patty Hermosilla, RN, and I support the concept.

New information appears to support careful use of probiotics in patients receiving antibiotics or for patients admitted to hospitals. Use of probiotics appears to either reduce the side effects of antibiotics (often GI tract) or help maintain healthy flora of $\mathrm{Gl}$ tract in hospitalized patients. Naturally occurring probiotics have been used by people over centuries (i.e. yogurt). Its used has increased all over the world but the research data is limited. More research will help clinicians understand potential application of probiotics in clinical practice.

Because of this, I support the research proposal.

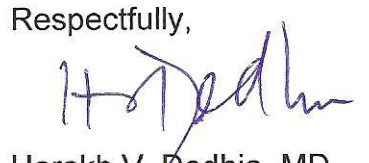

Harakh V. Dedhia, MD

Professor, Department of Medicine

Section of Pulmonary -CCM

WVU Health Science Center 


\section{Appendix C}

\section{WestVirginiaUniversityHospitals}

February 2, 2009

Patty Hermosilla, FNP-BC, MSN, RN

Senior Lecturer

Department of Health Restoration

West Virginia University, School of Nursing

PO Box 920

Morgantown, WV 26506-9620

Dear Patty:

I am writing this letter in support of the probiotic project. Probiotics are being used here at WVU in the critical care units in which I work, but most clinicians are not well informed about them. This project would help educate clinicians regarding the use of probiotics to prevent or decrease antibiotic-associated diarrhea. I think this would be a very worthwhile and needed study which would enable us to provide better care to our critically ill patients.

If I can be of assistance to you, please let me know.

Sincerely,

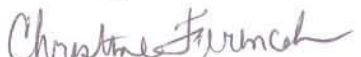

Christine Ferencak, RD 


\section{Appendix D}

\section{WestVrrginiaUniversityHospitals}

April 8, 2009

Patty Hermosilla, FNP-BC, MSN, RN

Senior Lecturer

Department of Health Restoration

West Virginia University, School of Nursing

PO BOX 9620

Morgantown, WV 26506-9620

\section{Dear Patty,}

This is to acknowledge receipt of your request in regards to the conduct of research entitled "Initiating a Practice Change: Prescribing Probiotics Concurrently with Antibiotic Therapy" to be conducted at West Virginia University Hospitals. My response is in the affirmative; you are granted permission to conduct your research, with the following stipulations:

1) Permission is granted based on the research being carried out precisely as defined in your methodology;

2) Permission is granted contingent upon approval and/or recommendations of the WVU Institutional Review Board:

3) At the completion of the study, you are requested to share your findings with those most concerned with the outcomes.

Best wishes to you in this endeavor!

Cordially,

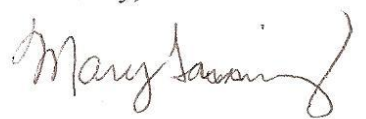

Mary Fanning, RN, MSN, CCRN, NEA-BC

Director, Nursing Administration

West Virginia University Hospitals

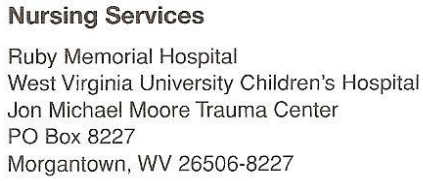




\title{
Appendix E
}

\author{
Guideline for Ordering a Probiotic
}

Patient is ordered an antibiotic.

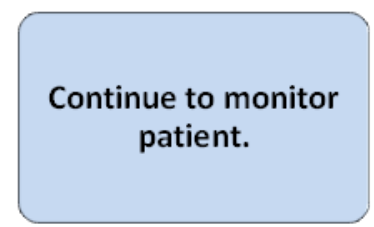

Is patient immune compromised? Does patient have short- gut syndrome, a non-

functioning Gl tract, or cardiac valve disease? Is yes STOP, if no continue.

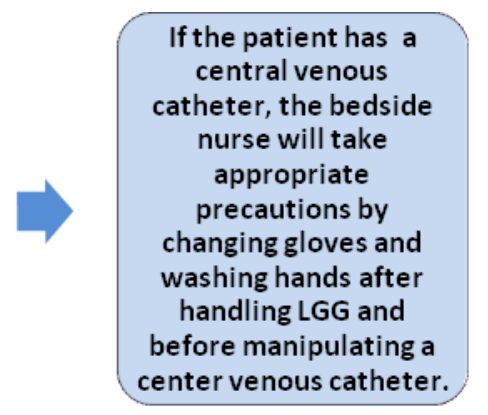

Order LGG $10^{10}$ CFU BID concurrently with antibiotic and for one week after completion of antibiotic. 


\section{Appendix F}

\section{Probiotic Survey}

\section{Check one:}

Physician

Nurse

How long have you been in practice?

For the following statements please answer according to the scale below: Thank you.

\begin{tabular}{|c|c|c|c|c|}
\hline $\begin{array}{c}\text { Strongly } \\
\text { Agree }\end{array}$ & Agree & Undecided & Disagree & $\begin{array}{c}\text { Strongly } \\
\text { Disagree }\end{array}$ \\
1 & 2 & 3 & 4 & 5 \\
\hline
\end{tabular}

Antibiotic Associated Diarrhea (AAD) is a significant health problem.

AAD can cause increased mortality, length of stay and cost of medical care for hospitalized patients.

There is sufficient evidence to support the routine administration of a probiotic concurrently with an antibiotic to reduce the occurrence and severity of AAD.

In most patients, probiotics are a simple, safe, cost effective method of decreasing the occurrence and severity of AAD.

I do not think probiotics are effective in reducing or preventing AAD. patients.

I believe the use of probiotics may be unsafe for many 
The following questions are designed to assess knowledge about probiotics and their use with antibiotics. Please answer to the best of your ability. If you do not know the answer, please indicate that you do not know rather than leaving the question blank. Please circle the correct answer or fill in the blank. Thank you for completing this questionnaire.

1. Which statements about probiotics are true?

a. Probiotics exhibit the same benefits, regardless of strain.

b. Probiotics exhibit strain-specific differences.

c. Probiotics are not of human origin.

d. Probiotics are affected by gastric acid and bile.

e. Unable to answer

2. The therapeutic dose of lactobacillus rhamnosus GG (LGG) is:
a. Once a day $10^{10}$ colony forming units
b. TID $10^{10}$ colony forming units
c. BID $10^{10}$ colony forming units
d. QID $10^{10}$ colony forming units
e. Unable to answer

3. What probiotics have the most level 1 supportive evidence (evidence derived from at least one properly designed randomized controlled trial)?
a. S. thermophilus and L. bulgaricus
b. Saccharomyces boulardii and lactobacillus GG
c. S. thermophilus and lactobacillus GG
d. lactobacillus $G G$ and L. bulgaricus
e. Unable to answer

4. Have you ever prescribed or recommended a probiotic to a patient (either from a food source or an OTC supplement)?

$$
\text { Yes No }
$$

5. If you answered "no" to \#4, do you have specific reservations about probiotics? 
6. Have you known people who have used probiotics effectively (either from a food source or supplement)?

Yes No

7. Have you personally used probiotics either in food sources or supplements?

Yes No

8. What might encourage you to prescribe or recommend probiotics?

9. What is the major patient risk factor for the development of AAD?
a. Poor general health
b. Poor nutrition
c. Age
d. Alteration in intestinal flora
e.Unable to answer

10. Antibiotic use has risen ㄷom 2002 to 2006 .
a. $1 \%$
b. $7 \%$
c. $25 \%$
d. $40 \%$
e.Unable to answer

11. In the last decade there has been a 응 increase in AAD.
a. $25 \%$
b. $50 \%$
c. $100 \%$
d. $500 \%$
e. Unable to answer 
12. The highest incidence of AAD is in:
a. The outpatient population
b. The intensive care unit
c. The pediatric population
d. The general medical unit
e. Unable to answer

13. What is the incidence of clostridium difficile acquisition among hospitalized patients?
a. 13\% in patients with hospital stays of up to two weeks and 50\% in those patient with hospital stay of up to four weeks
b. 30\% of all patients admitted to the hospital
c. 7\% in patients with hospital stays of up to two weeks and 20\% in those patient with hospital stay of up to four weeks
d. 25\% in patients with hospital stays of up to two weeks and 50\% in those patient with hospital stay of up to four weeks
e. Unable to answer

14. All antibiotics have the potential of causing AAD.

True False

15. Name three types of antibiotics that can reduce the number of gut anaerobes:

1 .

2 .

3 . 
16. Probiotics should be used with caution in patients who have/are: (check all that apply) immune compromised receiving multiply antibiotics short bowel syndrome central venous catheter elderly cardiac valve disease

17. How likely are you to prescribe or recommend a probiotic concurrently with an antibiotic?

Very likely

Somewhat likely

Neither likely nor unlikely

Somewhat unlikely

Unlikely 


\section{Appendix G}

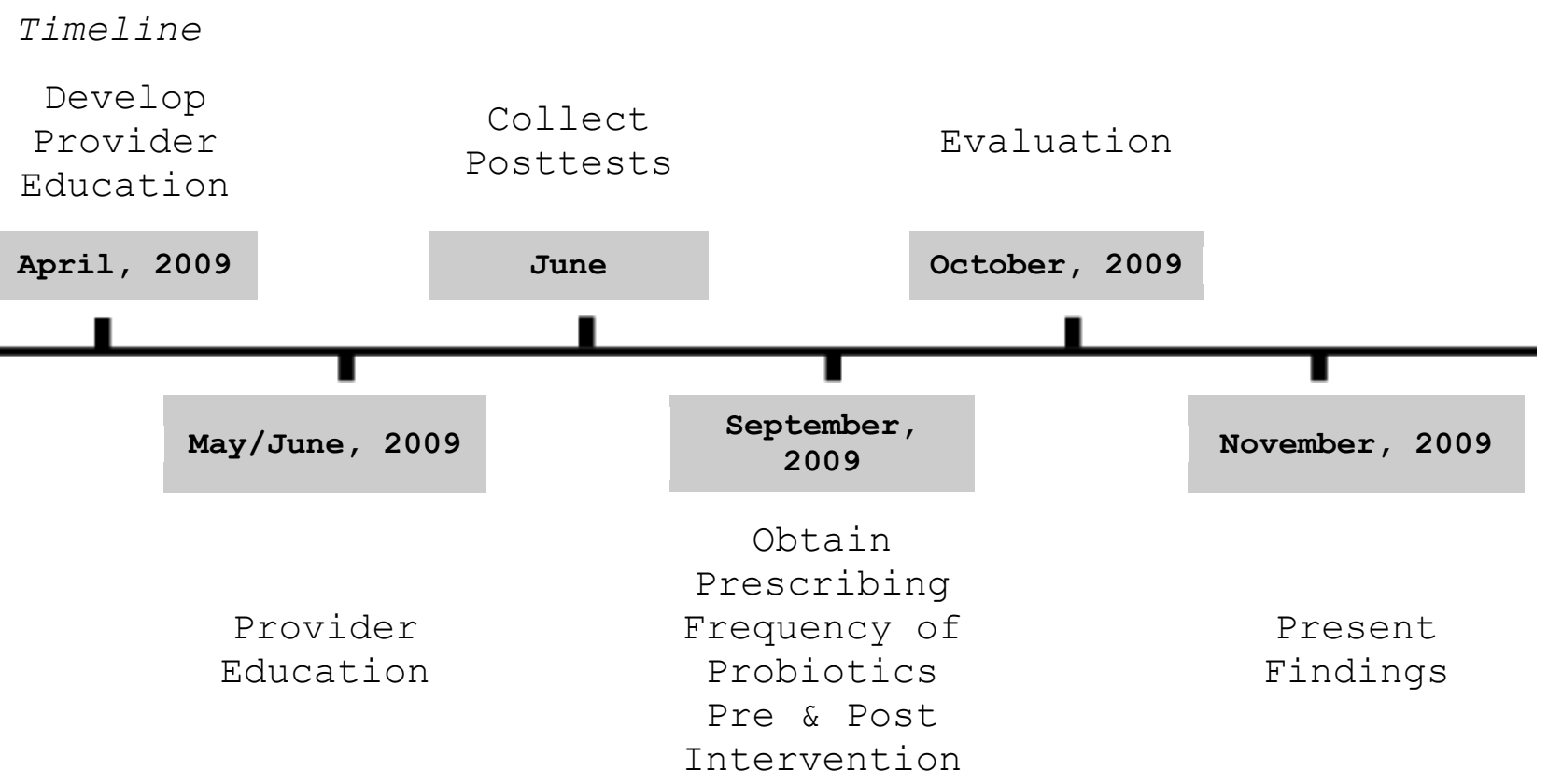




\section{References}

Beniwal, R.S., Arena, V. C. Thomas, L., Narla S., Imperiale, T., Chaudhry, R., et al. (2003). A randomized trial of yogurt for prevention of antibiotic-associated diarrhea. Digestive Diseases and Sciences, 48, 2077-2082.

Bergogne-Berezin, E. (2000). Treatment and prevention of antibiotic associated diarrhea. International Journal of Antimicrobial Agents, 16, 521-526.

Bernard, Claude (1927). Experimental Medicine. New Brunswick: Transaction Publishers.

Boyle, R. J., Robins-Browne, R. M., \& Tang, M. L. (2006). Probiotic use in clinical practice: what are the risks? American Journal of Clinical Nutrition, 83, 1256-1264.

Broussard, E. \& Surawicz, C. (2004). Probiotics and probiotics in clinical practice. Nutrition Clinical Care, 7, 104-113.

Cannon, W. B. (1939). The Wisdom of the Body ( $2^{\text {nd }}$ edition). New York: The Norton Library. 
Council for Agricultural Science and Technology (CAST), 2007. October). Probiotics: Their Potential to Impact Human Health. Issue Paper 36. Ames, Iowa.

Cedgard, L. (1998). Probiotics: The link between health and disease. Positive Health, 33, 39-43.

Chassany, O., Michaux, A. \& Bergmann, J. F. (2000). Drug-induced diarrhoea. Drug Safety 2000, 1, 53-72.

Cremonini, F., Di Caro, S., Nista, E. C., Bartolozzi, F., Capelli, G., Gasbarrini, G., et al. (2002). Meta-analysis: the effect of probiotic administration on antibioticassociated diarrhea. Aliment Pharmacology Therapy, 16, $1461-1467$.

D'Souza, A.S., Rajkumar, C. Cooke, J. \& Bulpitt, C. (2002). Probiotics in prevention of antibiotic associated diarrhea: meta-analysis. BMJ, 324,1-6.

Doron, S. I., Hibberd, P. L., \& Gorback, S. L. (2008). Probiotics for prevention of antibiotic-associated diarrhea. Journal of Clinical Gastroenterology, 42, (Supple 2) 58-63. 
Dunham, W. (2008, November 11). Hospitals use more antibiotics despite concerns. http://www. canada.com/story_print.html/id=950606\&sponsor=

Gill, H. S. \& Guarner, F., (2004). Probiotics and human health: a clinical perspective. Postgraduate Medical Journal, 80, $516-526$.

Hanaway, P., (2006). Balance of flora, galt, and mucosal integrity. Alternative Therapies, 12, 52-60.

Hawrelak, J.A., Whitten, D. L. \& Myers, S.P. (2005). Is lactobacillus rhamnosus GG effective in preventing the onset of antibiotic-associated diarrhoea: A systematic review. Digestion, 72, 51-56.

Hickson, M., D'Souza, A. L., Muthu, N, Rogers, T.R., Want, S.,Rajkumar, C. et al. (2007). Use of probiotic lactobacillus preparation to prevent diarrhea associated with antibiotics: randomized double blind placebo controlled trial BMJ, 1-5.

Hurley, B. W. \& Nguyen, C. C. (2002). The spectrum of pseudomembranous enterocolitis and antibiotic-associated diarrhea. Arch Intern Med, 162, 2177-2184. 
Joint FAO/WHO. (2002). Guidelines for the evaluation of probiotics in food, London Ontario, Canada, May 2002. Joint FAO/WHO Working Group: Author.

Koning, J.M., Jonkers, D., Stobberingh, E.E., Mulder, L., Rombouts, F.M. \& Stockbrugger, M.D.(2007). American Journal of Gastroenterology, 102, 1-12.

Lewin, Kurt (1997). Resolving social conflicts and Field theory in social science. Washington, DC: American Psychological Association

McFarland, L.V., (2006). Meta-Analysis of probiotics for the prevention of antibiotic associated diarrhea and the treatment of clostridium difficile disease. American Journal of Gastroenterology, 101, 812-822.

McVicar, A. \& Clancy, J. (1998). Homeostasis: a framework for integrating the life sciences. British Journal of Nursing, $7,601-607$

Meier, R. F. (2005) Probiotics: a new treatment for antibioticassociated diarrhea? Digestion 72, 49-50.

Ouwehand, A. C., Saxelin, M. \& Salimen, S. (2004). Phenotypic differences between commercial Lactobacillus rhamnosus GG 
and L. rhamnosus strains recovered from blood. Clinical Infectious Disease, 39, 1858-1860.

Pakyz, A. L., MacDougall, C., Oinonen, M. \& Polk, R. E. (2008). Trends in antibacterial use in US academic health centers. Arch Intern Med, 168, 2254-2260.

Penner, R., Fedorak, R. N., Madsen, K. L. (2005). Probiotics and nutraceuticals: non-medical treatments of gastrointestinal diseases. Current Opinion in Pharmacology, 5, 596-603.

Pestotnik, S. L., Classen D. C. Evans, R. C. \& Burke, J. P. (1996). Implementing antibiotic practice guidelines through computer-assisted decision support: clinical and financial outcomes. Annals of Internal Medicine, 124, 884-890.

Pham, M., Lemberg, D. A., Day, A. S. (2008). Probiotics: sorting the evidence from the myths. MJA, 188, 304-308.

Reid, G. \& Hammond, J. (2005). Probiotics some evidence of their effectiveness. Canadian Family Physician, 51, 1487-1493.

Reid, G., Jass, J., Subulsky, M. T., \& McCormick, J. K. (2003). Potential uses of probiotic in clinical practice. Clinical Microbiology Reviews, 16, 658-672. 
Reisinger, E. C., Fritzsche, C., Krause, R. \& Krejs, G. J. (2005). Diarrhea caused by primarily non-gastrointestinal infections. Nature Clinical Practice, 2, 216-222.

Rinomhota, A. S. \& Cooper, K. (1996). Homeostasis: restoring the internal wellbeing in patients/clients. British Journal of Nursing, 5, 1100-1108.

Salminen, M. K., Rautelin, H., Tynkkynen, S., Poussa, T., Saxelin, M., Valtonen, V., et al.(2004). Lactobacillus bactermia, clinical significance, and patient outcome, with special focus on probiotic L. Rhamnosus GG. Clinical Infectious Disease, 38, 62-69.

Schein, E. H. (1995). Kurt Lewin's change theory in the field and in the classroom: notes toward a model of managed learning. MIT Sloan School of Management.

Schroeder, M. S. (2005). Clostridium difficile-diarrhea. American Family Physician, 71, 921-928.

Sellin, J. H. (2001). The pathophysiology of diarrhea. Clinical Transplantation, 15, 2-10.

Synman, D. R. (2008). The safety of probiotics. Clinical Infectious Disease, 46(Suppl. 2), 104-111. 
Vanderhoff, J. A. \& Young, R. (2008). Probiotics in the United States. Clinical Infectious Disease, 46 (Suppl. 2), 67-72.

West Virginia University, Morgantown (2008) Retrieved April 9, 2008, from http://www.health.wvu.edu/general/Resources/missionstatements.aspx.

World Gastroenterology Organisation Practice Guideline. (2008, May). Probiotics and probiotics. World Health Organisation: Author. 